\title{
A Curriculum to Promote a Culture of Wellness Among Medical Students and Faculty
}

\author{
Catherine F. Pipas, MD, MPH | John A. Damianos, MD | Leah Montalbano, MPA | Allison L. \\ Matous, BA | Jianjun Hua, MS | Glenda H. Shoop, PhD
}

PRIMER. 2020;4:13.

Published: 7/10/2020 | DOI: 10.22454/PRiMER.2020.930805

\section{Abstract}

Introduction: Self-care has not been traditionally taught in medical education, but the epidemic of burnout among health professionals necessitates a change in culture, and consequently a change in curriculum. Burnout begins early in training and negatively impacts health professionals, patients, and institutions. Interventions that prevent and avert burnout are necessary at all stages of a doctor's career to assure wellbeing over a lifetime. Evidence-based strategies supporting both personal and system wellness have begun to emerge, but more research is needed.

Methods: We present a collaborative and comprehensive wellness program: "A Culture of Wellness." We offered this pilot jointly for first-year medical students and faculty volunteers at the Geisel School of Medicine at Dartmouth. We gave participants the following: (1) time-60 minutes per week for 8 weeks; (2) tools-weekly cases highlighting evidence-based wellness strategies; and (3) permission-opportunities to discuss and apply the strategies personally and within their community.

Results: Pre- and postsurvey results show that dedicated time combined with student-faculty collaboration and application of strategies was associated with significantly lower levels of burnout and perceived stress and higher levels of mindfulness and quality of life in participants. Components of the curriculum were reported by all to add value to personal well-being.

Conclusions: This pilot presents a feasible and promising model that can be reproduced at other medical schools and disseminated to enhance personal health and promote a culture of well-being among medical students and faculty.

\section{Introduction}

Quality of life and performance are significantly affected by burnout, which is prevalent in nearly $30 \%$ of the general population and over $50 \%$ of physicians. ${ }^{1}$ The emotional exhaustion, depersonalization, and loss of purpose that are characteristic of burnout exist across practice settings independent of gender, career stage, discipline, or specialty, and surface as early as the first year of medical school. ${ }^{1,2}$ Individuals experiencing burnout have lower job satisfaction, decreased quality of life, diminished work-life balance, and increased rates of substance use, depression, and suicide. ${ }^{3-5}$ Physician burnout is associated with increased medical errors and suboptimal patient care. ${ }^{6,7}$ Furthermore, health care systems bear increased costs and reduced productivity from job turnover, absenteeism, and presenteeism. ${ }^{8}$ Factors contributing to burnout exist at the individual, training environment, health care system, and society levels. ${ }^{1,9-11}$ 
Thomas Bodenheimer's Quadruple Aim challenged health care leaders to prioritize their health as a means to promote the health of others. ${ }^{12}$ Medical schools, residency programs, and clinical practices responded with evidence-based strategies and curricula for promoting personal and system well-being; however, the association with improved health is still limited. Further research is necessary. ${ }^{10,11,13-15}$

To combat the burnout epidemic, the Geisel School of Medicine offered a wellness program: "A Culture of Wellness." The purpose was to jointly augment wellness in students, faculty, and the medical school community by enhancing awareness, knowledge, skills, and application of personal and system-based health strategies to ultimately reduce levels of stress and burnout while improving quality of life and mindfulness.

\section{Methods}

After sending an open invitation to join the program through email, we held an introductory wellness session offering 20 slots ( 10 for first-year medical students and 10 for faculty). Program registration was offered on a firstcome basis. We considered all nonparticipating students and faculty to be the control group.

Participants were given time-they met together 60 minutes a week for 8 weeks; tools - they discussed weekly cases highlighting evidence-based wellness strategies; and permission-opportunities to examine and apply the strategies personally and within their community. Course objectives and participant commitments are listed in Table 1. Curriculum content and the weekly session format are listed in Table 2.

Assessment of the participants and control group examined changes in well-being and the potential impact of the curriculum. A pre- and postsurvey* was created to collect data in four areas: (1) quality of life, ${ }^{16}$ (2) perceived stress, ${ }^{17}$ (3) mindfulness, and (4) burnout. Questions used to assess mindfulness and burnout included, "Over the last month to what degree have you felt present in the moment?", and, "Over the last month have you felt burned out from your work?" We distributed the pre- and postsurveys to all first-year students and faculty regardless of participation. The postsurvey sent to participants asked additional questions regarding curriculum content and impact. To enable statistical analysis of responses, we assigned survey recipients numerical IDs prior to analysis to pair responses from pre- and postsurvey respondents. We limited access to recipient information to one study team member to ensure data integrity and confidentiality, and the methods of this pilot received IRB exemption as an educational improvement project.

\section{Results}

Ten first-year medical students, one second-year student facilitator, and 10 faculty members (including a facilitator) participated. All students and four faculty members completed the curriculum. Eleven completers across both groups submitted both a pre- and postsurvey.*

We conducted a paired $t$ test and we chose a statistical significance level (a) of $0.10(P<.10)$ as it reflects the nature of the pilot study, its small sample size, and unknown effect size. The small sample size resulting in low statistical power may limit the generalizability of the model established by the study, and the unknown effect size may cause uncertainty and potentially higher false positive rate. Therefore, we selected the significance level of 0.10 to acknowledge this pilot nature and warrant the potential effect in the study. We identified a statistically significant increase among participants' quality of life $(P=.03)$ and a statistically significant decrease in perceived stress $(P=.05)$. Using a 5-point Likert scale from not at all (1) to completely (5), a paired $t$ test showed a statistically significant increase in mindfulness $(P=.07)$. Results also indicated a $28 \%$ reduction in burnout (Table 3$)$. Presurvey, participants in the pilot study reported higher levels of burnout and stress and lower quality of life and mindfulness compared to the control group. Postsurvey, respondents expressed that the program gave them the permission and time needed to care for themselves, and several stated that the program encouraged them to be more authentic when interacting with colleagues (specific quotes are given in Table 4). All respondents agreed that cases, discussions, mindfulness exercises, and student-faculty collaboration completely or mostly added value to their well-being (Figure 1). Insufficient paired pre/postsurvey completion by the control group made pre/post comparison 
of the groups invalid.

\section{Conclusions}

The profession of medicine comes with occupational risks, ${ }^{13}$ and the epidemic of burnout in physicians presents a public health crisis negatively impacting physicians' health, patient care, and costs to health care systems. This pilot study proved a promising intervention to support students and faculty in augmenting personal and community wellness and a feasible preventative burnout program. Participants reported transformative changes resulting from the time, tools, and permission needed to prioritize wellness.

Time proved an asset and a challenge. Dedicated time to focus on wellness was valued by participants; however, lack of time hindered attendance. Paradoxically, permission to discuss the stigma of self-care and challenging culture contributed to successful collaborative interactions. Faculty willingness to share personal struggles allowed students to witness vulnerabilities rarely exposed. Faculty contributions illustrated that wellness is a perpetual journey of self-reflection and improvement. However, their attendance was also limited by time and competing priorities.

Limitations in addition to time include the small number of self-selected participants and low postsurvey response rate by faculty participants and the control group. Those attending the program may have been at higher risk for burnout and/or in need of well-being strategies. Of more concern is the possibility that those at highest risk and greatest need may not have participated. Within the small pilot study, we selected $P<.10$ as the threshold value, acknowledging that any significant effect in the results warrants, and indeed needs, further study before firm conclusions and generalizations can be made. This pilot study lays the groundwork for future extended and expanded study.

As medical educators, we are obligated to equip medical students and physicians alike with the knowledge, skills, and evidence-based well-being strategies to avert burnout and thrive in adversity. We are inspired to build on our successes and contribute to the broader needs of health professionals locally and nationally. Next steps include reassessing participants' schedules, designing incentives for survey completion, extending the program to the entire first-year class, and incorporating wellness competencies and metrics. We aim to disseminate our curriculum to other medical schools seeking to combat burnout with a wellness curriculum and welcome collaboration in the research.

\section{Footnote}

* Pre-Post Culture of Wellness Survey available at www.catherinefloriopipas.com.

\section{Tables and Figures}

Table 1: Course Description

\begin{tabular}{|l|l|}
\hline $\begin{array}{l}\text { Course } \\
\text { objectives }\end{array}$ & $\begin{array}{l}\text { Discuss the importance of wellbeing and the impact of burnout on individuals, teams, and communities; } \\
\text { Apply evidence-based wellness strategies for personal and community improvement; } \\
\text { Create and implement a personal health improvement plan. }\end{array}$ \\
\hline & $\begin{array}{l}\text { Attendance was requested for every session. Use of technology was permitted only as appropriate to } \\
\text { participation. Students and faculty participants were asked to read a weekly case, attend a } 60-\text { minute discussion } \\
\text { and participate as equals. They agreed to: (1) personal behavior that fosters respect, innovation, individual }\end{array}$ \\
$\begin{array}{l}\text { Participant } \\
\text { commitments } \\
\text { supportive, safe, and sustainable; (3) providing feedback that is constructive and promotes positive change; } \\
\text { and (4) upholding confidentiality. Participants and controls were given access to an electronic tool (Personal } \\
\text { Health Improvement Tool [PHIT] www.myphit.org), and asked to set a personal health priority, create a plan and } \\
\text { implement a project for personal improvement. Controls were given access only without support or monitoring. }\end{array}$ \\
\hline
\end{tabular}


Table 2: Course Format

\begin{tabular}{|l|l|}
\hline & $\begin{array}{l}\text { The Culture of Wellness curriculum was based on cases chosen from A Doctor's Dozen; 12 Strategies for } \\
\text { Personal Health and A Culture of Wellness. Cases are illustrated by patient stories, backed by science, and } \\
\text { linked to evidence-based wellness strategies and tools for personal application. Wellness strategies, listed } \\
\text { below, were chosen based on their applicability to the health professions. } \\
\text { Week 1- Meaning and Purpose } \\
\text { Week 2- Social Health and Connectivity } \\
\text { Week 3- Mindfulness and Self Reflection } \\
\text { Week 4- Resilience Training } \\
\text { Week 5- Emotional Intelligence and The Science of Change } \\
\text { Week 6- Time Management and Decision Making } \\
\text { Week 7- Cognitive Reframing and Affirmations } \\
\text { Week 8- Appreciative Inquiry and Gratitude }\end{array}$ \\
\hline $\begin{array}{l}\text { Welcome - Mindfulness Exercise (5 min). } \\
\text { - PHIT Check-in (10 min): Participants shared health improvement challenges and successes. } \\
\text { - Self-Reflection with Journaling (5 min): Participants were given a wellness journal and time to consider } \\
\text { facilitated questions prior to discussing. } \\
\text { - Facilitated Discussion of Strategy and Case (30 min): Three questions were presented weekly. The first } \\
\text { focused on the case and how the patient accessed the given tools and strategies to foster personal } \\
\text { health; the second on how participants could apply these same strategies personally; the third prioritized } \\
\text { utilizing the same tools to impact a culture of wellness. } \\
\text { format }\end{array} \quad \begin{array}{l}\text { Closing (5 min): Appreciative inquiry was used to express gratitude for sharing and feedback. Next session } \\
\text { logistics were reviewed. }\end{array}$ \\
\hline
\end{tabular}

Table 3: Pre/Postculture of Wellness Participant Outcomes

\begin{tabular}{|c|c|c|c|}
\hline & Pre & Post & Difference \\
\hline $\begin{array}{l}\text { Reduction in burnout } \\
\text { (Yes-No scale: percent of participants who selected "No") }\end{array}$ & $45 \%(5)$ & $73 \%(8)$ & $-28 \%$ \\
\hline $\begin{array}{l}\text { Reduction in perceived stress } \\
\text { (0-16 pt scale: Lower values reflect lower perceived stress) }\end{array}$ & 5.9 & 4.3 & $-1.6(P=.052)$ \\
\hline $\begin{array}{l}\text { Increase in quality of life } \\
\text { (0-60 pt scale: higher values reflect higher quality of life) }\end{array}$ & 40.4 & 46.1 & $5.7(P=.028)$ \\
\hline $\begin{array}{l}\text { Increase in mindfulness } \\
\text { (1-5 pt Likert scale: higher values reflect higher mindfulness) }\end{array}$ & 2.8 & 3.5 & $0.7(P=.066)$ \\
\hline
\end{tabular}

Table 4: Participant Quotes

\footnotetext{
- "The program gave me permission to make time for things that bring me joy \& [sic] replenish me during this marathon of a career in medicine."

- "I will reframe more, I will take time to take care of myself, I will teach others to meditate."

- "I will try to convey a deeper level of genuine care when asking "how are you?" to peers and will respond to their greetings with more honesty."

- "I will be actively listening and curious about others [sic] wellbeing. I will share with others and be vulnerable so we change this culture of fear and burnout"

- "The Culture of Wellness program has made juggling everything so much more manageable. I saw a huge difference in my ability to cope with finals this semester compared to last not only in terms of studying but also in terms of keeping things balanced with my family and my general wellness. I am excited for the opportunity to keep the program going later on this year."

- "This class helped me appreciate the privilege and the challenges of medical school and has encouraged me to pursue the "nonmedical" things I love without guilt. I know maintaining wellness is a long, perhaps never complete process, and one I hope I can successfully continue in the years ahead."
} 
Figure 1: Value Added of Culture of Wellness Program Components

\section{Culture of Wellness: \\ To what degree did the following components add value to your well-being?}

Weekly facilitated case discussion on strategies for personal health and a culture of wellness

Weekly 5 min mindfulness exercise to begin each session

Weekly hour with peers and faculty on wellness topics

Presence of faculty and 2nd year as colleagues in discussions

Weekly "check in" on MyPHIT (Roses and Thorns)

Weekly Reading/Cases

Formal time for personal reflection/journalling at each session

Dinner with peers

Social time with 4th year HSP students

Use of the www.MyPHIT.org website

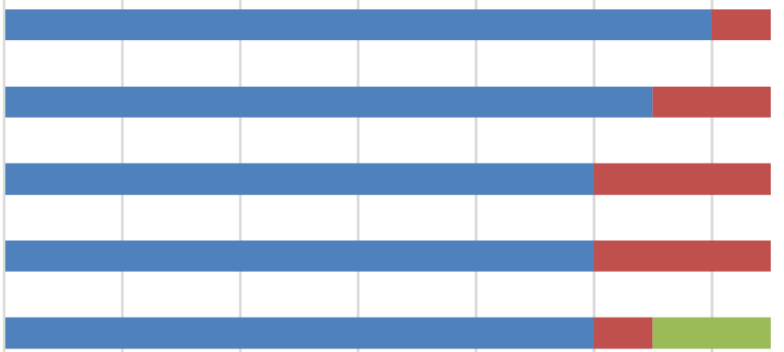

(1)

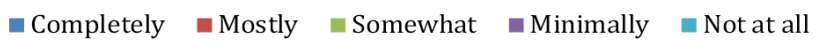

*Only 11 of the 13 participants rated the value of "Dinner with peers" and "Social time with fourth-year HSP students" as they indicated they did not attend.

\section{Acknowledgments}

Acknowledgment: The authors thank participants in this program for caring for themselves and contributing to our growing culture of wellness.

Presentations: Components and strategies of the curriculum were presented at the STFM Medical Student Education Conference, Austin, Texas. Dr Pipas was the Plenary Speaker, Cura te Ipsum "Physician Heal Thyself"; Strategies for Modeling and Sustaining Personal and Systems Wellness. Dr Pipas also led the following workshop at the same meeting:

Pipas, CF, Cur ate Ipsum "Heal Thyself": Strategies to Avert Burnout, Build Resilience and Model Wellness" Conference on Medical Student Education, Feb. 2018 Austin, Texas.

Conflict Disclosure: Dr Pipas is the author of A Doctor's Dozen: 12 Strategies for Personal Health and A Culture of Wellness.

\section{Corresponding Author}

Leah Montalbano, MPA

Geisel School of Medicine at Dartmouth, Remsen Rm 310, Hanover, NH 03755. 603-650-1187. Fax: 603-650-1251.

Leah.Montalbano@Dartmouth.edu

\section{Author Affiliations}

Catherine F. Pipas, MD, MPH - Geisel School of Medicine, Dartmouth College, Hanover, NH

John A. Damianos, MD - Yale New Haven Hospital 
Leah Montalbano, MPA - Geisel School of Medicine, Dartmouth College, Hanover, NH

Allison L. Matous, BA - Geisel School of Medicine, Dartmouth College, Hanover, NH

Jianjun Hua, MS - Dartmouth College, Hanover, NH

Glenda H. Shoop, PhD - Geisel School of Medicine, Dartmouth College, Hanover, NH

\section{References}

1. Shanafelt TD, Hasan O, Dyrbye LN, et al. Changes in burnout and satisfaction with work-life balance in physicians and the general US working population between 2011 and 2014. Mayo Clin Proc. 2015;90(12):1600-1613. doi:10.1016/j.mayocp.2015.08.023

2. Dyrbye LN, West CP, Satele D, et al. Burnout among U.S. medical students, residents, and early career physicians relative to the general U.S. population. Acad Med. 2014;89(3):443-451. doi:10.1097/ACM.0000000000000134

3. Rotenstein LS, Ramos MA, Torre M, et al. Prevalence of depression, depressive symptoms, and suicidal ideation among medical students: a systematic review and meta-analysis. JAMA. 2016;316(21):2214-2236. doi:10.1001/jama.2016.17324

4. Erschens R, Keifenheim KE, Herrmann-Werner A, et al. Professional burnout among medical students: systematic literature review and meta-analysis. Med Teach. 2019;41(2):172-183.

doi:10.1080/0142159X.2018.1457213

5. Ishak W, Nikravesh R, Lederer S, Perry R, Ogunyemi D, Bernstein C. Burnout in medical students: a systematic review. Clin Teach. 2013;10(4):242-245. doi:10.1111/tct.12014

6. Shanafelt TD, Balch CM, Bechamps G, et al. Burnout and medical errors among American surgeons. Ann Surg. 2010;251(6):995-1000. doi:10.1097/SLA.0b013e3181bfdab3

7. West CP, Dyrbye LN, Shanafelt TD. Physician burnout: contributors, consequences and solutions. J Intern Med. 2018;283(6):516-529. doi:10.1111/joim.12752

8. Dewa CS, Loong D, Bonato S, Thanh NX, Jacobs P. How does burnout affect physician productivity? A systematic literature review. BMC Health Serv Res. 2014;14(1):325. doi:10.1186/1472-6963-14-325

9. Peckham C. Medscape National Physician Burnout \& Depression Report, 2018. https://www.medscape.com /slideshow/2018-lifestyle-burnout-depression. Accessed March 13, 2018.

10. Dzau VJ, Kirch DG, Nasca TJ. To care is human - collectively confronting the clinician-burnout crisis. N Engl J Med. 2018;378(4):312-314. doi:10.1056/NEJMp1715127

11. National Academies of Sciences, Engineering, and Medicine. Taking Action Against Clinician Burnout: A Systems Approach to Professional Well-Being. Washington, DC: The National Academies Press; 2019.

12. Bodenheimer T, Sinsky C. From triple to quadruple aim: care of the patient requires care of the provider. Ann Fam Med. 2014;12(6):573-576. doi:10.1370/afm.1713

13. Shanafelt TD, Noseworthy JH. Executive leadership and physician well-being: nine organizational strategies to promote engagement and reduce burnout. Mayo Clin Proc. 2017;92(1):129-146.

doi:10.1016/j.mayocp.2016.10.004

14. Shanafelt T, Trockel M, Ripp J, Murphy ML, Sandborg C, Bohman B. Building a program on well-being: key design considerations to meet the unique needs of each organization. Acad Med. 2019;94(2):156-161. doi:10.1097/ACM.0000000000002415

15. Wasson LT, Cusmano A, Meli L, et al. Association between learning environment interventions and medical student well-being: a systematic review. JAMA. 2016;316(21):2237-2252. doi:10.1001/jama.2016.17573

16. Thomas MR, Dyrbye LN, Huntington JL, et al. How do distress and well-being relate to medical student empathy? A multicenter study. J Gen Intern Med. 2007;22(2):177-183. doi:10.1007/s11606-006-0039-6

17. Warttig SL, Forshaw MJ, South J, White AK. New, normative, English-sample data for the short form perceived stress scale (PSS-4). J Health Psychol. 2013;18(12):1617-1628. doi:10.1177/1359105313508346 